\title{
Radiating stars with generalised Vaidya atmospheres
}

\author{
S. D. Maharaj • G. Govender • M. \\ Govender
}

Received: date / Accepted: date

\begin{abstract}
We model the gravitational behaviour of a radiating star when the exterior geometry is the generalised Vaidya spacetime. The interior matter distribution is shear-free and undergoing radial heat flow. The exterior energy momentum tensor is a superposition of a null fluid and a string fluid. An analysis of the junction conditions at the stellar surface shows that the pressure at the boundary depends on the interior heat flux and the exterior string density. The results for a relativistic radiating star undergoing nonadiabatic collapse are obtained as a special case. For a particular model we demonstrate that the radiating fluid sphere collapses without the appearance of the horizon at the boundary.
\end{abstract}

Keywords heat conducting fluids · radiating stars · relativistic astrophysics

\section{Introduction}

The study of radiating stars in the context of general relativity has generated much interest in researchers because of the variety of applications in relativistic astrophysics. These studies are important as they enable us to investigate physical features such as surface luminosity, dynamical stability, particle production at the stellar surface, relaxation effects, causal temperature gradients and other thermodynamical processes. Some relevant references investigating these issues are given by Di Prisco et al. [1, Govender et al. [2, Herrera et al. [3] and Pinheiro and Chan 4. Relativistic radiating stars are also important in the process of gravitational collapse, describing the final state of stars, formation of singularities and black hole physics, in four and higher dimensions. Recent investigations in this regard are contained in the works of Goswami and Joshi [5], Joshi [6] and Madhav et al. 7]. In particular, the validity of the cosmic censorship conjecture can be tested in this physical scenario.

S. D. Maharaj · G. Govender · M. Govender

Astrophysics and Cosmology Research Unit, School of Mathematical Sciences, University of KwaZulu-Natal, Private Bag X54001,Durban 4000, South Africa

E-mail: maharaj@ukzn.ac.za 
The model of a relativistic radiating star undergoing dissipation was completed by Santos [8] by analysing the junction conditions at the stellar surface. By matching a shear-free interior spacetime to the radiating Vaidya exterior spacetime, he showed that at the surface the pressure is nonvanishing and proportional to the heat flux. Subsequently several explicit relativistic radiating stellar models have been found by investigating the appropriate boundary condition. Kramer [9] and Maharaj and Govender [10] generated nonstatic radiating spheres from a static model by allowing certain parameters to become functions of time. Kolassis et al. [1] and Thirukkanesh and Maharaj [12] assumed geodesic fluid trajectories to produce new radiating models. In the approach of De Oliviera et al. 13 and Nogueira and Chan 14 the model has an initial static configuration before the radiating sphere starts gradually to collapse. Exact solutions for shear-free interiors which are conformally flat generate radiating stellar models as shown by Herrera et al. [15], Herrera et al. [16], Maharaj and Govender [17] and Misthry et al. [18. Stellar models which are radiating with nonzero shear are difficult to analyse because of the complexity of the boundary condition. However even in this case there have been advances in obtaining exact solutions. Particular exact models have been found by Naidu et al. [19], Rajah and Maharaj [20] and Pinheiro and Chan [21].

In this paper we seek to generalise the Santos junction conditions by matching a shear-free interior spacetime to the generalised Vaidya exterior spacetime. The energy momentum tensor of the generalised Vaidya spacetime may be interpreted as a superposition of two fluids, a null dust and a null string fluid. The physical properties of the generalised Vaidya spacetime have been discussed by Husain 22 ] and Wang and $\mathrm{Wu}[23$. Glass and Krisch 24 have interpreted the exterior spacetime as a superposition of two fluids outside a relativistic star, the original Vaidya null fluid and a new null fluid composed of strings. By assuming diffusive transport for the string fluid Glass and Krisch 25] found new solutions to Einstein's equations with transverse stresses. Physically reasonable energy transport mechanisms have been generated by Krisch and Glass 26 in the stellar interior with the generalised Vaidya metric as the exterior spacetime. These investigations, and other treatments, have largely focussed on physical processes in the exterior of the stellar model with a generalised Vaidya atmosphere. To fully describe a radiating stellar model requires generation of the junction conditions at the stellar surface.

We follow the convention that the coupling constant $\frac{8 \pi G}{c^{4}}$ and the speed of light $c$ are unity; the metric has signature $(-+++)$. In Sect. 2 we present the field equations for the interior and exterior spacetimes. In Sect. 3 the matching of the interior and exterior spacetimes across the stellar surface is outlined. The new set of junction conditions are derived for the generalised Vaidya spacetime. We indicate how the new junction conditions generalise the junction conditions previously derived by Santos 8. The physical significance of our new result is highlighted in terms of a string fluid. We also consider the new junction condition in the context of conservation of momentum flux across the stellar boundary. In Sect. 4 we generate a particular model and demonstrate that the new generalised junction condition has a solution. In this model it is possible for the radiating fluid sphere to collapse without the appearance of a boundary. The results of this paper are briefly summarised in Sect. 5 . 


\section{Field Equations}

Spacetime needs to be divided into two distinct regions, the interior spacetime $\mathcal{M}^{-}$and the exterior spacetime $\mathcal{M}^{+}$for a stellar model. The boundary of the star $\Sigma$ serves as the matching surface for $\mathcal{M}^{-}$and $\mathcal{M}^{+}$. The boundary or stellar surface is a timelike three-dimensional hypersurface. We require for the first junction condition that

$$
\left(d s_{+}^{2}\right)_{\Sigma}=\left(d s_{-}^{2}\right)_{\Sigma}=d s_{\Sigma}^{2}
$$

so that the line elements match on the boundary $\Sigma$. The second junction condition is generated by the continuity of the extrinsic curvature of $\Sigma$ across the boundary given by

$$
\left(K_{\alpha \beta}^{+}\right)_{\Sigma}=\left(K_{\alpha \beta}^{-}\right)_{\Sigma}
$$

Note that the junction conditions (11) and (2) are equivalent to the Lichnerowicz [27] and O' Brien and Synge 28] junction conditions.

The line element for the interior manifold $\mathcal{M}^{-}$is given by

$$
d s^{2}=-A^{2}(t, r) d t^{2}+B^{2}(t, r)\left[d r^{2}+r^{2}\left(d \theta^{2}+\sin ^{2} \theta d \phi^{2}\right)\right]
$$

in comoving and isotropic coordinates. The interior spacetime is expanding and accelerating but is shear-free. A physically relevant interior matter distribution that is consistent with (3) is given by

$$
T_{a b}^{-}=(\mu+p) u_{a} u_{b}+p g_{a b}+q_{a} u_{b}+q_{b} u_{a}
$$

where $\mu$ is the energy density, $p$ is the isotropic pressure, $q_{a}$ is the radial heat flux vector and $u^{a}=\frac{1}{A} \delta_{0}^{a}$ is the comoving fluid four-velocity. The Einstein field equations $G_{a b}^{-}=T_{a b}^{-}$for the interior manifold $\mathcal{M}^{-}$are given by

$$
\begin{aligned}
\mu= & 3 \frac{\dot{B}^{2}}{A^{2} B^{2}}-\frac{1}{B^{2}}\left(2 \frac{B^{\prime \prime}}{B}-\frac{B^{\prime 2}}{B^{2}}+\frac{4}{r} \frac{B^{\prime}}{B}\right) \\
p= & \frac{1}{A^{2}}\left(-2 \frac{\ddot{B}}{B}-\frac{\dot{B}^{2}}{B^{2}}+2 \frac{\dot{A}}{A} \frac{\dot{B}}{B}\right) \\
& +\frac{1}{B^{2}}\left(\frac{B^{\prime 2}}{B^{2}}+2 \frac{A^{\prime}}{A} \frac{B^{\prime}}{B}+\frac{2}{r} \frac{A^{\prime}}{A}+\frac{2}{r} \frac{B^{\prime}}{B}\right) \\
p= & -2 \frac{\ddot{B}}{A^{2} B}+2 \frac{\dot{A}}{A^{3}} \frac{\dot{B}}{B}-\frac{\dot{B}^{2}}{A^{2} B^{2}}+\frac{1}{r} \frac{A^{\prime}}{A B^{2}} \\
& +\frac{1}{r} \frac{B^{\prime}}{B^{3}}+\frac{A^{\prime \prime}}{A B^{2}}-\frac{B^{\prime 2}}{B^{4}}+\frac{B^{\prime \prime}}{B^{3}} \\
q= & -\frac{2}{A B^{2}}\left(-\frac{\dot{B}^{\prime}}{B}+\frac{B^{\prime} \dot{B}}{B^{2}}+\frac{A^{\prime}}{A} \frac{\dot{B}}{B}\right)
\end{aligned}
$$

where dots and primes denote differentiation with respect to $t$ and $r$ respectively. The results (1) -(5) were first obtained by Santos [8].

The line element for the exterior manifold $\mathcal{M}^{+}$is taken to be

$$
d s^{2}=-\left(1-2 \frac{m(v, \mathrm{r})}{\mathrm{r}}\right) d v^{2}-2 d v d \mathrm{r}+\mathrm{r}^{2}\left(d \theta^{2}+\sin ^{2} \theta d \phi^{2}\right)
$$


where $m(v, r)$ is the mass function, and is related to the gravitational energy within a given radius $r$ (Lake and Zannias [29, Poisson and Israel [30]). This metric is often called the generalised Vaidya spacetime since it reduces to the Vaidya spacetime when $m=m(v)$ which is the mass of the star as measured by an observer at infinity. It has been demonstrated by Husain [22] and Wang and Wu [23] that an energy momentum tensor consistent with (6) is

$$
\begin{aligned}
T_{a b}^{+} & =T_{a b}^{(n)}+T_{a b}^{(m)} \\
& =\varepsilon l_{a} l_{b}+(\rho+P)\left(l_{a} n_{b}+l_{b} n_{a}\right)+P g_{a b}
\end{aligned}
$$

which represents a superposition of a null dust and a null string fluid. In general $T_{a b}^{+}$represents a Type II fluid as defined by Hawking and Ellis [31. The null vector $l^{a}$ is a double null eigenvector of the energy momentum tensor $T_{a b}^{+}$. The weak and strong energy conditions, and the dominant energy conditions are satisfied for proper choices of the mass function $m(v, r)$. In (7) we have introduced the two null vectors

$$
l_{a}=\delta_{a}^{0}, \quad n_{a}=\frac{1}{2}\left[1-2 \frac{m(v, r)}{r}\right] \delta_{a}^{0}+\delta_{a}^{1}
$$

where $l_{a} l^{a}=n_{a} n^{a}=0$ and $l_{a} n^{a}=-1$. The Einstein field equations $G_{a b}^{+}=T_{a b}^{+}$ for the exterior manifold $\mathcal{M}^{+}$are then given by

$$
\begin{aligned}
\varepsilon & =-2 \frac{m_{v}}{\mathrm{r}^{2}} \\
\rho & =2 \frac{m_{\mathrm{r}}}{\mathrm{r}^{2}} \\
P & =-\frac{m_{\mathrm{rr}}}{\mathrm{r}}
\end{aligned}
$$

where we have used the notation

$$
m_{v}=\frac{\partial m}{\partial v}, \quad m_{\mathrm{r}}=\frac{\partial m}{\partial \mathrm{r}}
$$

We interpret $\varepsilon$ as the density of the null dust radiation; $\rho$ and $P$ are the null string density and null string pressure, respectively. Note that (7)- (9c) were derived by Wang and $\mathrm{Wu}[23$.

\section{Generalised Santos conditions}

It is possible to match the spacetimes (3) and (6) across the boundary $\Sigma$. Since the derivation is similar to the Santos [8] treatment we provide only an outline of the argument for our more general case with $m=m(v, \mathrm{r})$. The intrinsic metric to the hypersurface $\Sigma$ is defined by

$$
d s_{\Sigma}^{2}=-d \tau^{2}+\mathcal{Y}^{2}\left(d \theta^{2}+\sin ^{2} \theta d \phi^{2}\right)
$$

For the interior spacetime $\mathcal{M}^{-}$we obtain

$$
\begin{aligned}
A\left(r_{\Sigma}, t\right) d t & =d \tau \\
r_{\Sigma} B\left(r_{\Sigma}, t\right) & =\mathcal{Y}(\tau)
\end{aligned}
$$


For the exterior region $\mathcal{M}^{+}$we generate the results

$$
\begin{aligned}
\mathrm{r}_{\Sigma}(v) & =\mathcal{Y}(\tau) \\
\left(1-\frac{2 m}{\mathrm{r}}+2 \frac{d \mathbf{r}}{d v}\right)_{\Sigma} & =\left(\frac{d v}{d \tau}\right)_{\Sigma}^{-2}
\end{aligned}
$$

Equations (11) and (12) correspond to the first junction condition (11). Observe that the quantity $\tau$ was defined on the surface $\Sigma$ as an intermediate variable. On eliminating $\tau$ we have

$$
\begin{aligned}
A\left(r_{\Sigma}, t\right) d t & =\left(1-\frac{2 m}{\mathrm{r}_{\Sigma}}+2 \frac{d \mathrm{r}_{\Sigma}}{d v}\right)^{1 / 2} d v \\
\mathrm{r}_{\Sigma}(v) & =r B\left(\mathrm{r}_{\Sigma}, t\right)
\end{aligned}
$$

Equations (13) are the necessary and sufficient conditions for the first junction condition (1) to be valid.

The intrinsic curvature for the interior spacetime $\mathcal{M}^{-}$has the form

$$
\begin{aligned}
& K_{11}^{-}=\left(-\frac{1}{B} \frac{A^{\prime}}{A}\right)_{\Sigma} \\
& K_{22}^{-}=\left[r(r B)^{\prime}\right]_{\Sigma} \\
& K_{33}^{-}=\sin ^{2} \theta K_{22}^{-}
\end{aligned}
$$

The extrinsic curvature for the exterior spacetime $\mathcal{M}^{+}$has the form

$$
\begin{aligned}
K_{11}^{+} & =\left[\frac{\tilde{\tilde{v}}}{\tilde{v}}-\tilde{v} \frac{m}{\mathrm{r}^{2}}+\tilde{v} \frac{m_{\mathrm{r}}}{\mathrm{r}}\right]_{\Sigma} \\
K_{22}^{+} & =\left[\tilde{v}\left(1-\frac{2 m}{\mathrm{r}}\right) \mathrm{r}+\mathrm{r} \tilde{\mathrm{r}}\right]_{\Sigma} \\
K_{33}^{+} & =\sin ^{2} \theta K_{22}^{+}
\end{aligned}
$$

where $\tilde{\mathrm{r}}=\frac{d \mathrm{r}}{d \tau}$ and $\tilde{v}=\frac{d v}{d \tau}$. Observe the appearance of the term containing $m_{\mathrm{r}}$ in $K_{11}^{+}$which does not exist in the treatment of Santos [8]. As we shall see later this has a profound effect on the physics of the model. Equations (14) and (15) correspond to the second junction condition (2). The mass profile in terms of the metric functions can be generated by eliminating $r, \tilde{r}$ and $\tilde{v}$. We observe that

$$
m(v, r)=\left[\frac{r B}{2}\left(1+r^{2} \frac{\dot{B}^{2}}{A^{2}}-\frac{1}{B^{2}}\left(B+r B^{\prime}\right)^{2}\right)\right]_{\Sigma}
$$

which is the total gravitational energy contained within the stellar surface $\Sigma$. We also establish the relationship

$$
\begin{gathered}
\left(-\frac{1}{B} \frac{A^{\prime}}{A}\right)=\left(1+r \frac{B^{\prime}}{B}+r \frac{\dot{B}}{A}\right)^{-1} \\
\times\left[\frac{m_{\mathrm{r}}}{\mathrm{r}}+\frac{B^{\prime}}{B^{2}}+\frac{r}{2} \frac{B^{\prime 2}}{B^{3}}-\frac{r}{2} \frac{\dot{B}^{2}}{B A^{2}}-\frac{1}{A}\left(r \frac{\dot{B}^{\prime}}{B}+r \frac{\ddot{B}}{A}-r \frac{B^{\prime} \dot{B}}{B^{2}}-r \dot{B} \frac{\dot{A}}{A^{2}}\right)\right]_{\Sigma}
\end{gathered}
$$


This expression may be simplified further: multiply with $1+r \frac{B^{\prime}}{B}+r \frac{\dot{B}}{A}$ and utilise $(5 b)$ and $(5 d)$. We then arrive at the result

$$
p=\left(q B-2 \frac{m_{r}}{r^{2} B^{2}}\right)_{\Sigma}
$$

which generalises the junction condition of Santos [8]. Hence we have demonstrated that the junction conditions (2) are equivalent to

$$
\begin{aligned}
m(v, \mathrm{r}) & =\left[\frac{r B}{2}\left(1+r^{2} \frac{\dot{B}^{2}}{A^{2}}-\frac{1}{B^{2}}\left(B+r B^{\prime}\right)^{2}\right)\right]_{\Sigma} \\
p & =\left(q B-2 \frac{m_{\mathrm{r}}}{\mathrm{r}^{2}}\right)_{\Sigma}
\end{aligned}
$$

Equations (19) are the necessary and sufficient conditions for the second junction condition (2) to be valid. We point out that the mathematical approach and procedure that we have followed is similar to Santos 8]. However in our case the external stellar atmosphere is the generalised Vaidya spacetime. The form of the equations (10)-(19b) can be related to the equations of Santos 8 since they have a similar structure. The fact that $m=m(v, r)$ fundamentally affects the final result. The equations for the extrinsic curvature (15a), the matching condition (17) and junction condition (18) are fundamentally different.

We have generated the relationships (13) and (19) so that the junction conditions (11) and (2) are satisfied for the shear-free interior spacetime (3) and the generalised Vaidya exterior spacetime (6) across the hypersurface $\Sigma$. This generalises the Santos [8] result for a relativistic radiating star when $m=m(v)$. Observe that when $m$ depends on the coordinate $v$ only then (19b) becomes

$$
p=q B
$$

at the boundary $\Sigma$, which is the earlier Santos junction condition. When (20) is valid then the pressure $p$ on the boundary depends only on the heat flux $q$. We have shown here that if $m=m(v, r)$ then $(19 b)$ is valid, and the pressure $p$ on the boundary depends on the heat flux $q$ and the gradient $m_{\mathrm{r}}(v, \mathrm{r})$.

The generalised Vaidya spacetime has physical significance and contains many known solutions of the Einstein field equations with spherical symmetry. It contains the monopole solution, the de Sitter and Anti-de Sitter solutions, the charged Vaidya solution and the radiating dyon solution. The physical features and the energy momentum complexes, that provide acceptable energy momentum distributions for these systems, have been studied by Barriola and Vilenkin [32, Chamorro and Virbhadra [33, Virbhadra [34]-36] and Yang [37. Glass and Krisch [24]-25] and Krisch and Glass [26] have interpreted the generalised Vaidya spacetime to represent a superposition of an atmosphere composed of two fluids: a string fluid and a null dust fluid. This atmosphere may model several physical situations at different distance scales, eg. the exterior regions of black holes (distance scale of multiples of the Schwarzschild radius) and globular clusters containing a component of dark matter (distance scale of the order of parsecs). The additional term $2 \frac{m_{r}}{r^{2}}$ in the boundary condition $(19 b)$ arises from the matching at the surface $\Sigma$. This quantity has physical significance and can be interpreted as a particular 
contribution from the energy momentum tensor. We observe that the term $2 \frac{m_{\mathrm{r}}}{\mathrm{r}^{2}}$ in $(19 b)$ is the same quantity as that in (9b). Therefore we may interpret the quantity $2 \frac{m_{r}}{r^{2}}$ as the string density $\rho$.

We can therefore write (19b) in the more transparent form

$$
p=[q B-\rho]_{\Sigma}
$$

at the boundary $\Sigma$. Consequently for a radiating star with outgoing dissipation in the form of radial heat flow, with the generalised Vaidya spacetime as the exterior, the pressure on the surface depends on the interior heat flux $q$ and the exterior string density $\rho$. The appearance of the quantity $\rho$ in (21) allows for more general behaviour that was the case in the Santos 8] treatment. From (20) we observe that $q=0$ implies that $p=0$ on $\Sigma$ and the exterior manifold $\mathcal{M}^{+}$must be the Schwarzschild exterior metric with $m$ being constant. In (21) we note that we obtain the Schwarzschild exterior geometry when $q=0=\rho$. However it is clear from (21) that when $q B=\rho$ then $p=0$ on $\Sigma$ and the exterior spacetime remains the generalised Vaidya spacetime with $m=m(v, r)$. In addition, when $q=0$ then $p=-\rho$ on $\Sigma$ and the interior is not radiating.

It is possible to provide a physical interpretation of our result by consideration of the momentum flux across the boundary $\Sigma$. Since the quantity (19a) represents the total gravitational energy for a sphere of radius $r$ within $\Sigma$ we can write $m(v, r)=m(t, r)$. Partially differentiating (19a) eventually leads to the result

$$
p_{\Sigma}=-\frac{2}{r^{2}} \tilde{v}^{2} m_{v}-\frac{2}{r^{2}} m_{\mathrm{r}}
$$

which reduces to the corresponding Santos [8] equation when $m=m(v)$. The radial flux of momentum across the hypersurface $\Sigma$ is defined by

$$
F^{ \pm}=e_{0}^{ \pm a} n^{ \pm b} T_{a b}^{ \pm}
$$

where $e_{0}^{ \pm a}$ and $n^{ \pm b}$ are vectors which are respectively tangent and normal to $\Sigma$. For conservation of momentum flux across $\Sigma$ we must have

$$
F^{+}=F^{-}
$$

In the interior manifold $\mathcal{M}^{-}$we can generate the quantity

$$
F^{-}=-q B
$$

In the exterior manifold $\mathcal{M}^{+}$we can produce the quantity

$$
F^{+}=\frac{2}{r^{2}} \tilde{v}^{2} m_{v}
$$

Then equations (22)- (26) yield the result

$$
p_{\Sigma}=\left(q B-\frac{2}{r^{2}} \frac{m_{\mathrm{r}}}{B^{2}}\right)_{\Sigma}
$$

which is the same as (19b). Therefore the junction condition (19b) corresponds to the conservation of the radial flux of momentum across the hypersurface $\Sigma$. It represents the local conservation of momentum. 


\section{A particular model}

To illustrate the utility of the generalised Santos condition we consider a specific example relating to horizons. For simplicity we take the mass function to be of the form $m=\tilde{p} r^{2}$ where $\tilde{p}$ is an arbitrary constant. For this example we consider a particular form of the metric coefficients given in (3). We choose the coefficients to be separable in $r$ and $t$ so that

$$
A=a(r), \quad B=b(r) R(t)
$$

The field equations (5) then yield the quantities

$$
\begin{aligned}
& \mu=\frac{1}{R^{2}}\left[\frac{3}{a^{2}} \dot{R}^{2}-\frac{1}{b^{2}}\left(\frac{2 b^{\prime \prime}}{b}-\frac{b^{\prime 2}}{b^{2}}+\frac{4 b^{\prime}}{r b}\right)\right] \\
& p=\frac{1}{R^{2}}\left[-\frac{1}{a^{2}}\left(2 R \ddot{R}+\dot{R}^{2}\right)+\frac{1}{b^{2}}\left(\frac{b^{\prime 2}}{b^{2}}+\frac{2 a^{\prime} b^{\prime}}{a b}+\frac{2}{r}\left(\frac{a^{\prime}}{a}+\frac{b^{\prime}}{b}\right)\right)\right] \\
& q=-\frac{2 a^{\prime} \dot{R}}{R^{3} a^{2} b^{2}}
\end{aligned}
$$

and the condition for the isotropy of pressure

$$
\frac{a^{\prime \prime}}{a}+\frac{b^{\prime \prime}}{b}-2 \frac{b^{\prime 2}}{b^{2}}-2 \frac{a^{\prime} b^{\prime}}{a b}-\frac{a^{\prime}}{r a}-\frac{b^{\prime}}{r b}=0
$$

The boundary condition (19b) now yields at $r=r_{\Sigma}$ the equation

$$
2 R \ddot{R}+\dot{R}^{2}+\mathcal{M} \dot{R}=\mathcal{N}+\mathcal{P}
$$

where we have used the particular choice of the mass function $m=\tilde{p} r^{2}$, the potentials (27) and the system (28). In the above we have set

$$
\begin{aligned}
\mathcal{M} & =-\frac{2 a^{\prime}}{b} \\
\mathcal{N} & =\frac{a^{2}}{b^{2}}\left[\frac{b^{\prime 2}}{b^{2}}+2 \frac{a^{\prime} b^{\prime}}{a b}+\frac{2}{r}\left(\frac{a^{\prime}}{a}+\frac{b^{\prime}}{b}\right)\right] \\
\mathcal{P} & =6 \frac{a^{2} \tilde{p}}{b^{2}}
\end{aligned}
$$

which are constants in the integration process as (30) holds on the boundary $\Sigma$.

Equation (30) does not have a general solution in closed form; for the purpose of our investigation we observe that it admits an elementary particular solution of the form

$$
R(t)=-\mathcal{C} t
$$

where $\mathcal{C}$ is given by

$$
\mathcal{C}=\frac{1}{2}\left[\mathcal{M} \pm\left(\mathcal{M}^{2}+4(\mathcal{N}+\mathcal{P})\right)^{\frac{1}{2}}\right]
$$


This solution is useful in that at the surface of the collapsing star the ratio $\frac{m_{\Sigma}}{r_{\Sigma}}$ is independent of time. The mass profile given by (19a) and the solution (32) yield the following expression

$$
\frac{2 m_{\Sigma}}{\mathrm{r}_{\Sigma}}=2\left[\frac{r^{2} b^{2} \mathcal{C}^{2}}{2 a^{2}}-\frac{r b^{\prime}}{b}-\frac{r^{2} b^{\prime 2}}{2 b^{2}}\right]_{\Sigma}
$$

which is valid on the stellar surface. It is clear that the quantities in the above equation are evaluated at the boundary $\Sigma$; there is sufficient freedom provided by $r, a, b$ and $\mathcal{C}$ so that the right hand side can be constrained in such a manner that the ratio $\frac{2 m_{\Sigma}}{r_{\Sigma}}$ is strictly less than unity. Consequently no horizon will form. We can illustrate this explicitly by setting $b(r)=1$ and $a(r)=\frac{1}{2} \xi r^{2}+\beta$ where $\beta$ and $\xi$ are arbitrary constants. Then we get

$$
1-\frac{2 m_{\Sigma}}{r_{\Sigma}}=\left[1-\frac{\mathcal{C}^{2} r^{2}}{\left(\beta+\frac{1}{2} \xi r^{2}\right)^{2}}\right]_{\Sigma}
$$

We choose $\mathcal{C}^{2}$ to be less than $\frac{\beta^{2}}{r^{2}}+\beta \xi+\frac{1}{4} \xi^{2} r^{2}$ on $\Sigma$ so that $1-\frac{2 m_{\Sigma}}{r_{\Sigma}}$ is not zero for all time. Consequently the boundary surface never reaches the horizon. This simple example demonstrates the absence of the horizon and is similar to the result of Banerjee et al. [38] which holds in the conventional Vaidya spacetime. We regain their results when $\mathcal{P}=0$ in (31). We interpret our result to mean that there is no accumulation of energy in the interior of the radiating star as it releases energy at the rate of generation during the collapse.

\section{Conclusion}

In this work we have produced a general model of a relativistic radiating star by performing the smooth matching of a shear-free interior spacetime to the generalised Vaidya exterior spacetime, across a timelike spatial hypersurface. We have demonstrated that with the generalised Vaidya radiating metric, the junction conditions on the stellar surface change substantially, and consequently represents a more general atmosphere surrounding the star. The atmosphere is a superposition of null dust and a string fluid. We find that the density of the string fluid affects the pressure at the stellar boundary. We have shown explicitly that

$$
p=q B-\rho_{\text {string }}
$$

at the stellar surface. If the weak and strong energy conditions or the dominant energy conditions are satisfied then $\rho_{\text {string }} \geq 0 \quad(\mu \neq 0)$ and $\rho_{\text {string }} \geq P_{\text {string }} \geq$ $0 \quad(\mu \neq 0)$ respectively. This indicates that for outgoing heat flux in gravitational collapse, the exterior string fluid density reduces the pressure on the stellar boundary. It is interesting to note that we have shown using a geometric approach that the derivative of the mass function with respect to the exterior radial coordinate is related to the string density. By means of a simple example we have considered the formation of horizons for a heat conducting sphere which radiates energy into the generalised Vaidya external atmosphere during collapse. The horizon does not appear in this example at any stage of the collapse. 
Acknowledgements MG and GG thank the National Research Foundation and the University of KwaZulu-Natal for financial support. SDM acknowledges that this work is based upon research supported by the South African Research Chair Initiative of the Department of Science and Technology and the National Research Foundation. The authors would also like to thank Sanjay Wagh and Subharthi Ray for constructive criticisms and useful discussions.

\section{References}

1. Di Prisco, A., Herrera, L., Le Denmat, G., MacCallum, M.A.H., Santos, N.O.: Phys. Rev. D 76, $064017(2007)$

2. Govender, M., Maharaj, S. D., Maartens, R.: Class. Quantum Grav. 15, 323 (1998)

3. Herrera, L.,Le Denmat, G., Santos, N.O.: Phys. Rev. D 79, 087505 (2009)

4. Pinheiro, G., Chan, R.: Gen. Relativ. Gravit. 40, 2149 (2008)

5. Goswami, R., Joshi, P.S.: Phys. Rev. D 76, 084026 (2007)

6. Joshi, P.S.: Pramana - J. Phys. 69, 119 (2007)

7. Madhav, T.A., Goswami, R., Joshi, J.S.: Phys. Rev. D 72, 084029 (2005)

8. Santos, N.O.: Mon. Not. R. Astron. Soc. 216, 403 (1985)

9. Kramer, D.: J. Math. Phys. 33, 1458 (1992)

10. Maharaj, S. D., Govender, M.: Aust. J. Phys. 50, 959 (1997)

11. Kolassis, C.A., Santos, N.O., Tsoubelis, D.: Astrophys. J. 327, 755 (1988)

12. Thirukkanesh, S., Maharaj, S.D.: J. Math. Phys. 50, 022502 (2009)

13. de Oliviera, A.K.G., Santos, N.O, Kolassis, C.A.: Mon. Not. R. Astron. Soc. 216, 1001 (1985)

14. Nogueira, P.C., Chan, R.: Int. J. Mod. Phys. D 13, 1727 (2004)

15. Herrera, L., Le Denmat, G., Santos, N.O., Wang, A.: Int. J. Mod. Phys. D 13, 583 (2004)

16. Herrera, L., Di Prisco, A., Ospino, J.: Phys. Rev. D 74, 044001 (2006)

17. Maharaj, S.D., Govender, M.: Int. J. Mod. Phys. D 14, 667 (2005)

18. Misthry, S.S., Maharaj, S.D., Leach, P.G.L.: Math. Meth. Appl. Sci. 31, 363 (2008)

19. Naidu, N.F., Govender, M., Govinder, K.S.: Int. J. Mod. Phys. D 15, 1053 (2006)

20. Rajah, S.S., Maharaj, S.D.: J. Math. Phys. 49, 012501 (2008)

21. Pinheiro, G., Chan, R.: Gen. Relativ. Gravit. 43, 1451 (2011)

22. Husain, V.: Phys. Rev. D 53, 1759 (1996)

23. Wang, A., Wu,Y.: Gen. Relativ. Gravit. 31, 107 (1999)

24. Glass, E.N., Krisch, J.P.: Phys. Rev. D 57, 5945 (1998)

25. Glass, E.N., Krisch, J.P.: Class. Quantum Grav. 16, 1175 (1999)

26. Krisch, J.P., Glass, E.N.: J. Math Phys. 46, 062501 (2005)

27. Lichnerowicz, A.: Theories Relativistes de la Gravitation et de l'Electromagnetisme. Masson, Paris (1955)

28. O' Brien, S., Synge, J.L.: Dublin Inst. Adv. Stud. A 9, 1 (1952)

29. Lake, K., Zannias, T.: Phys. Rev. D 43, 1798 (1991)

30. Poisson, E., Israel, W.: Phys. Rev. D 41, 1796 (1990)

31. Hawking, S.W., Ellis, G.F.R.: The large scale structure of spacetime. Cambridge University Press, Cambridge (1973)

32. Barriola, M., Vilenkin, A.: Phys. Rev. Lett. 63, 341 (1989)

33. Chamorro, A., Virbhadra, K.S.: Pramana - J. Phys. 45, 181 (1995)

34. Virbhadra, K.S.: Phys. Rev. D 41, 1086 (1990)

35. Virbhadra, K.S.: Phys. Rev. D 42, 2919 (1990)

36. Virbhadra, K.S.: Phys. Rev. D 60, 104041 (1999)

37. Yang, I.C.: Chin. J. Phys. 45, 497 (2007)

38. Banerjee, A., Chatterjee, S., Dadhich, N.K.: Mod. Phys. Lett. A 17, 2335 (2002). 